\title{
Radiation Therapy Moves on Several Fronts
}

Aside from GSI's commitment to set up a high-energy ion therapy unit, there have recently been some other significant milestones in the field of radiotherapy based on heavy charged particles offering treatment of tumours and brain malformations while sparing surrounding tissue close to critical organs. The Editor reviews European developments.

\section{HIGH-ENERGY PROTON THERAPY}

Ion beam Applications SA (IBA), Louvain-laNeuve: The Massachusetts General Hospital (MGH), in Boston, USA, has carried out proton therapy on the largest number of patients (some 6000). In February, the MGH selected a consortium headed by IBA to supply a complete, 850 patients per year, high-energy (235 MeV) proton cyclotron-based unit for its Northwest Proton Therapy Center. This represents the first commercial order for a dedicated accelerator for hospital-based medical applications. It includes beam lines, isocentric gantries for rotating the beam around the patient, and patient positioners allowing both passive beam spreading and pencil-beam scanning.

Cyclotron Research Centre (CRC), Louvainla-Neuve: IBA grew out of cyclotron-based activities at the University of Louvain's CRC, which is one of the rare institutions to equip itself with three successive generations of cyclotrons. Protons of up to $95 \mathrm{MeV}$ are supplied by the $110 \mathrm{MeV}$ isochronous cyclotron CYCLONE for treating ocular and other shallow melanomas. The Centre plans to improve its patient handling facilities but does not envisage a major high-energy proton therapy unit, preferring to leave this to several hospital-based projects now being studied in Belgium.

Paul Scherrer Institute (PSI), Zurich: The PSI has treated in its OPTIS facility the second largest number of patients (over 1500 with ocular melanomas) using $72 \mathrm{MeV}$ protons from an injector cyclotron. PSI will start operating this summer Europe's first high-energy proton radiotherapy unit to use a dedicated isocentric gantry (it has a novel, compact design). Deep-seated tumours will be irradiated with a $250 \mathrm{MeV}$ degraded beam from the Institute's ring cyclotron. A proton radiosurgery unit for brain treatment with a few$\mathrm{mm}$ in diameter beam has been proposed as a replacement for PSI's 60-beam, pion-therapy unit PIOTRON which was shut down in 1992 owing to difficulties with the complex cryogenic system.
Hahn-Meitner Institute (HMI), Berlin: The HMI in collaboration with eye specialists from the Free University's Steglitz Clinic, is likely to receive approval this year for federal and local funding for a $2.3 \mathrm{MDM}$ facility to treat ocular melanomas with up to $72 \mathrm{MeV}$ protons coming from its separate-sector cyclotron VICKSI (using $30 \%$ of 3 weeks/month beam time). Helium ions can be accelerated, but the energy would be too low so the HMI has no immediate plans for extending therapy activities.

Centre Antoine Lacassagne, Nice: The Centre's Cyclotron Laboratory MEDICYC has treated the eyes of about 350 patients with $65 \mathrm{MeV}$ protons. A $200 \mathrm{MeV}$ whole-body proton therapy unit based on a compact, isocentric gantry has also been designed. The Centre collaborated with Siemens in designing a compact superconducting cyclotron, but the company is presently re-examing its involvement and has no definite commercial plans at this stage.

Institut de Physique Nucléaire (IPN), Orsay: The IPN has treated the eyes of about 350 patients (using protons from a $200 \mathrm{MeV}$ synchrotron). Moving to higher energies to treat brain tumours using an isocentric gantry is planned, but funding is so far undefined.

Kernfysisch Versneller Institute (KVI), Groningen: The KVI will take delivery this summer of its new $200 \mathrm{MeV}$ superconducting cyclotron AGOR. AGOR was constructed at the IPN, Orsay, and it achieved its first main goal on 12 April by producing a beam from each of its four extraction channels; the official dedication is on 27 May. A proposed proton therapy facility is presently being discussed with funding agencies.

Forschungszentrum Jülich (KFA): The KfK has proposed a whole-body $200 \mathrm{MeV}$ proton therapy facility using a beam from its $40-2500$ $\mathrm{MeV}$ Cooler-Synchrotron COSY (it would in principle be able to move to nitrogen or oxygen ions). The KFA's Board of Directors recently indicated that it cannot move ahead right now so the plan is to start experiments in physics-related and other aspects for a few years. One advantage is the presence on-site of a 40-bed clinic belonging to Düsseldorf University.

Clatterbridge Centre for Oncology, Wirral: Proton therapy using a $62 \mathrm{MeV}$ cyclotron at the Douglas Cyclotron Unit is firmly established (500 patients treated). A proposa within about one year, possibly in collaboration with AEA Technology, Harwell, is expected in order to boost the cyclotron's energy to $200 \mathrm{MeV}$, mainly for brain therapy.

\section{LIGHT-ION RADIOTHERAPY}

Heavy-Ion Medical Accelerator (HIMAC), Chiba, Japan: The world's first, large, accelerator-based light-ion therapy facility to be built for purely medical purposes treated its first patient last month. It is based on a pair of $41 \mathrm{~m}$ in diameter synchrotrons that can accelerate ions from helium (often misleadingly called a heavy ion) to argon up to 800 $\mathrm{MeV} /$ nucleon. The design permits simultaneous irradiation using horizontal and vertical beams, and explains in part the high capital and operating costs ( $300 \mathrm{MS}$ and $50 \mathrm{~K} \$$ / patient, respectively) which have conveyed the mistaken impression that light-ion therapy is inherently expensive.

Istituto Nazionale di Fisica Nucleare (INFN): The INFN launched Progetto Adroterapia (Hadrontherapy Project) in 1991 aiming at setting up the most advanced tumour radiation therapy techniques in Italy. An epidemiological survey has indicated that the best solution would be several regional therapy facilities, each equipped with compact accelerators and a large (1000 patients/year) centre. A subsequent study recommended that the centre should use $250 \mathrm{MeV}$ protons from a $\mathrm{H}^{-}$synchrotron. A possible second stage envisages light-ion (carbon and oxygen) therapy at $400 \mathrm{MeV} /$ nucleon. A detailed feasibility study ends in July.

\section{AUSTRON: Austria's proposed AUSTRON} spallation neutron source [see EN 24 (1993) 156] aims to provide light-ion therapy at 400-500 MeV/nucleon. A feasibility study nearing completion envisages a first stage involving research, with a separate project for a therapy centre for routine treatment requiring installation of a gantry and possibly a storage ring.

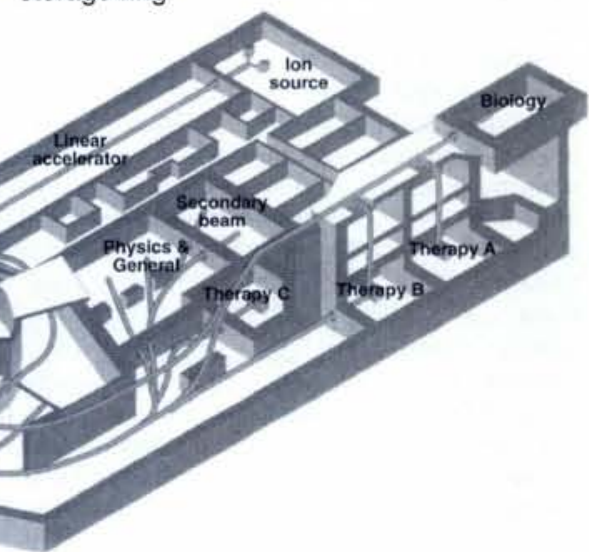

The HIMAC facility in Chiba on the outskirts of Tokyo. A pair of $41 \mathrm{~m}$ in diameter synchrotrons provides lightion beams to three treatment rooms.
The Editor wishes to thank the following for their Vervier (Louvain), H. Homeyer (HMI), R. Maier $(\mathrm{KFI}), \mathrm{G}$. Kraft (GSI), A. Kacperek (Clatterbridge), U. Amaldi (CERN and Milan). comments: P. Mandrillon (Nice), R. André (IBA), J.
A cut-away view of the Northeast Proton Therapy Center showing treatment rooms each with an isocentric gantry 